\title{
Tokamak equilibria with toroidal current reversal: properties and computational issues
}

\author{
Paulo Rodrigues and João P. S. Bizarro \\ Centro de Fusão Nuclear, Associação Euratom-IST, Instituto Superior Técnico, 1049-001 \\ Lisboa, Portugal.
}

\begin{abstract}
Several properties of axisymmetric plasma equilibria with toroidal-current reversal (TCR) are discussed, using some unifying concepts from catastrophe theory [1]. Namely, those of structural stability of functions near critical points, singularity unfolding by small perturbations, and model parameter-space division by bifurcation sets are found to be of particular usefulness. Magnetic configurations displaying, simultaneously, TCR and nested flux surfaces are thence shown to be necessarily degenerate and structurally unstable, meaning that they are easily transformed into non-nested ones by small perturbations in the model parameter set. This should lead to a new paradigm when discussing TCR equilibria, as most of present knowledge relies mainly on the properties of nested solutions [2, 3, 4]. It is thus expected to favor the study of the broader class of non-nested configurations, which has recently attracted a considerable discussion in the fusion community $[5,6,7]$. In addition, it is also shown how TCR imposes some constraints on plasma profiles, and how these may be dealt with computationally while keeping the ability to manipulate the shape of the inner island system.
\end{abstract}

\section{REFERENCES}

1. R. Gilmore, Catastrophe theory for scientists and engineers, John Wiley and Sons, New York, 1981.

2. M. S. Chu, and P. B. Parks, Phys. Plasmas 9, 5036-5042 (2002).

3. G. W. Hammett, S. C. Jardin, and B. C. Stratton, Phys. Plasmas 10, 4048-4052 (2003).

4. Y. I. Pozdnyakov, Phys. Plasmas 12, 084503 (2005).

5. A. A. Martynov, S. Y. Medvedev, and L. Villard, Phys. Rev. Lett. 91, 085004 (2003).

6. S. Wang, Phys. Rev. Lett. 93, 155007 (2004).

7. P. Rodrigues, and J. P. S. Bizarro, Phys. Rev. Lett. 95, 015001 (2005). 